\title{
PARA UNA ECONOMÍA POLÍTICA DEL CONOCIMIENTO DESDE EL SUR GLOBAL: CONDICIONES, MÉTODO, CONEXIONES
}

\author{
Jorge Daniel Vásquez \\ Pontificia Universidad Católica del Ecuador/ \\ Universidad de Massachusetts-Amherst \\ jdvasquez@puce.edu.ec
}

Recibido: 1 de mayo de 2020 / Aceptado: 7 de mayo de 2020 / Publicado: 19 de junio de 2020

\section{Resumen}

A partir de algunos aspectos en el pensamiento de Marx, se trabajan conceptos que han acompañado el análisis de la economía política, y se problematizan las condiciones y el método para una economía política del conocimiento. También se plantean las conexiones necesarias para un análisis desde el Sur Global. Así, este artículo se refiere a la devaluación de la fuerza de trabajo en los patrones de acumulación del capital y en las formas de desposesión en el siglo XXI, además de incorporar la crítica a las perspectivas eurocéntricas.

Palabras clave: Capitalismo, conocimiento, economía política, eurocentrismo, Sur Global

\begin{abstract}
Addressing some aspects of Marx's thought, it not only works on concepts that have accompanied the analysis of the political economy but also problematizes the conditions and method for a political economy of knowledge, as well as the necessary connections for an analysis from the Global South. Thus, considering the devaluation of the labor force and the expansion of dispossession in 21st-century capitalist accumulation, the article incorporates criticism of Eurocentric perspectives.
\end{abstract}

Key Words: Capitalism, Knowledge, Political Economy, Eurocentrism, Global South

Este artículo contiene algunos elementos teóricos a ser considerados en una economía política del conocimiento desde el Sur Global. A este respecto, se describen tres aspectos cruciales que constituyen la base de un proyecto de este tipo. En primer lugar, se señala un conjunto de condiciones en las que debe situarse al análisis de la producción del conocimiento que tiene que ver 
con los procesos de acumulación por desposesión (una forma particular de la acumulación primitiva) que, aunque la doctrina político-económica neoliberal se remonta a la década de 1930, se hizo más notoria desde mediados de 1970. En segundo lugar, se refiere al Método de economía política para señalar hasta qué punto el análisis de la producción del conocimiento puede ser susceptible a la lectura particular de categorías de Marx. Esta sección se refiere a la Historia económica de la producción del conocimiento tal como la ha planteado el historiador Joel Mokyr de frente a un marco crítico para analizar las sociedades capitalistas del siglo XXI. Finalmente, se plantea la relación entre los caminos del desarrollo capitalista en el Sur Global (centrándose en América Latina y África).

\section{Acumulación capitalista y devaluación de la fuerza de trabajo: Condiciones}

La economía política del conocimiento requiere un análisis dedicado a establecer las relaciones que se forman en torno a la generación y la apropiación de excedente en el proceso de producción del conocimiento. Este análisis es relevante debido a que en el siglo XXI la formación social dada posee el conocimiento como una fuente significativa de valor. No obstante, este proceso no está exento de las contradicciones del capitalismo y de su lógica inherente de erosión de las condiciones de producción. Al observar los efectos sociales, ecológicos y culturales sobre las poblaciones del Sur Global y las condiciones precarias de los trabajadores en el Norte Global, la economía política del conocimiento puede estar situada en el centro de la "acumulación por desposesión” imperialista (Harvey, 2003: 137-182).

Pensar desde el Sur Global requiere considerar la coproducción dialéctica del sujeto del conocimiento (es decir, los trabajadores o la fuerza de trabajo, el Estado o el conjunto de instituciones) y cómo opera el nuevo imperialismo a través de formas particulares de acumulación durante el neoliberalismo. Desde la década de 1970, los activos en todo el mundo, que anteriormente estaban bajo propiedad colectiva (es decir, por parte del Estado o en común), se han visto forzados al ámbito del mercado a través de fuerzas violentas o coercitivas (y donde el Estado y el capital privado han trabajado juntos).

Al seguir el argumento de Harvey, la crisis de sobreacumulación ocurre cuando los excedentes de capital y de mano de obra no pueden tomar 
su rentabilidad para lograr un poder socialmente útil, o, en palabras de Harvey, "overaccumulation is a condition where surplus of capital (perhaps accompanied by surplus of labor) lie idle with no profitable outlets in sight" (2003, 149). Tal crisis puede derivar en una de estas dos opciones, o en una combinación de ambas: expansión geográfica y reorganización espacial (lo que implica no solo una infraestructura física de larga vida sino también social). En este sentido, Harvey argumenta que, desde mediados de la década de 1970, el capitalismo ha experimentado la incapacidad de acumular a través de la reproducción expandida.

El análisis que hace Harvey (2003) sobre el desarrollo capitalista se basa en el concepto de "acumulación primitiva”, como Karl Marx ([1867]2009) lo propone en El Capital (Vol I, parte VIII). Por lo tanto, Harvey (2003, 145152) argumenta que la acumulación primitiva es la base de toda acumulación capitalista posterior y que, para que el capitalismo la sostenga, esta debe ser reiterada. En el capitalismo contemporáneo, esta forma de acumulación por desposesión no trata solo de superar la crisis de sobreacumulación, sino que también es la forma dominante en el sistema global.

La acumulación por desposesión libera un conjunto de bienes o de mano de obra a un costo exiguo (o a costo cero). Esto significa que el capital sobreacumulado se apodera de esos activos y los convierte en un uso rentable, tal como Marx describió en El Capital (cap. VIII)-los cercamientos que expulsaron a los residentes para crear proletariado sin tierra, liberando la tierra en la tendencia dominante de privatización. Sin embargo, desde mediados de la década de 1970, el neoliberalismo ha recreado el proceso de privatización a través de la liberación de materias primas baratas en el sistema y la devaluación de los activos de capital existentes y de la fuerza laboral. El capital financiero y el Estado vinculan la acumulación por desposesión y la reproducción expandida (Harvey lo llama "el cordón umbilical”). El análisis empírico de casos particulares en el Sur Global muestra que muchas formas de propiedad pública han sido mercantilizadas, mientras que la mercantilización del trabajo se ha intensificado, al incluir la proletarización del campesinado y los pueblos indígenas (Chatterjee 2016; Alimonda, Toro y Martín 2017; Tijoux 2016).

Por otro lado, la acumulación por desposesión puede considerarse una expresión del imperialismo. Para Harvey, la acumulación por desposesión aparece no solo en la periferia del sistema mundial capitalista, sino también en el 
centro pues la relación entre estos dos está dentro del marco del imperialismo. Para Rosa Luxemburgo ([1913]1951), el capitalismo siempre debe tener algo fuera de sí para su proceso de estabilización y encontrar soluciones temporales a su crisis, es decir, la imposición de la acumulación primitiva en la periferia. Harvey sigue esta idea y argumenta que la acumulación por desposesión no es exclusiva de la periferia, aunque es más inhumana en lo que llamamos el Sur Global, o "in regions within uneven geographical development" (Harvey 2003, 173).

Así, la acumulación por desposesión es la manifestación de un "Nuevo Imperialismo". En el nuevo imperialismo (es decir, las relaciones sociales organizadas capitalistas desde los años 70), la devaluación de la fuerza de trabajo es posible precisamente debido a la crisis, que funciona como una ola previa de devaluación, y a través de la cual el capitalismo perpetuamente crea su otro periférico.

En los países industrializados, el papel dado a la ciencia y al conocimiento después de la Segunda Guerra Mundial se consolidó a finales del siglo XX (Meyer y Ramírez (2010, 235). Además, con la victoria liberal, se censuraron y estigmatizaron los ataques contra determinados grupos étnicos, religiosos y nacionales. Estas visiones generales, basadas en principios universales comunes a todos los individuos explican, por ejemplo, el auge de las ciencias médicas en las universidades, que se basaba en el valor universal de la vida y del cuerpo humano. El desarrollo científico en las universidades se expandió junto con una visión global del conocimiento, combinada con procedimientos de diferenciación en cuanto al lugar y valor de éstos por fuera de la academia del Norte Global (Vásquez, Cielo y Bravo 2016).

Los patrones históricos del siglo XX tienen sentido no solo desde una perspectiva económica, sino también desde el campo de las relaciones de poder político y la hegemonía en el nivel mundial (Vakulabharanam 2014). Esta combinación de las transformaciones económicas y la configuración histórico-política de la hegemonía debe analizarse en dos niveles: los períodos de acumulación estable a mediano plazo (que siempre tienen lugar solo unas pocas décadas) y los períodos de tiempo más prolongados en los que una configuración espacial es hegemónica dentro de una estructura central y periférica (de los Países Bajos a Gran Bretaña, de Gran Bretaña/Estados Unidos a China). 
Las crisis se refieren a períodos de interrupción severa del proceso "normal" de reproducción capitalista e implican un estancamiento durante muchos años (Wright 1978). Por lo tanto, es necesaria una perspectiva dialéctica en la que los elementos que se utilizan para solventar ciertas crisis sean considerados como los que pueden causar una crisis particular en un futuro régimen (Harvey 1982; Vakulabharanam 2014). El carácter dialéctico que Marx utilizó para su método también parece ser la naturaleza del capitalismo, ya que este es una relación en movimiento, o una relación que de acuerdo a los momentos históricos es mutable (Vakulabharanam 2014). De aquí que, para abordar la cuestión del conocimiento en este escenario, se torne necesario volver al problema del método.

\section{Economía política del conocimiento: Método}

El destacado historiador Joel Mokyr (2002) argumenta que una economía política del conocimiento se refiere al análisis de la innovación técnica y la resistencia a la misma. Mokyr argumenta que el elemento central en la producción de conocimiento es su "utilidad", ya sea como conocimiento proposicional (es decir, creencias sobre fenómenos naturales y regularidades), o como conocimiento prescriptivo (esto es, técnicas). Así, para Mokyr, la economía política del conocimiento es el análisis de la tensión histórica con respecto a la producción e implementación del conocimiento útil en las sociedades (Mokyr 2002, 3-15; 218-283).

Los elementos de la concepción de Mokyr giran en tríada dentro de la transformación tecnológica de las sociedades occidentales:

Historically, a technological choice is made whenever a new technique is proffered, and selectors (firms and households) have to decide whether to adopt it. It might seem that in the vast majority of cases, this decision is trivial: if the new technique increases efficiency and profits it will be adopted; otherwise, it will not. However, few economies have ever left these decisions entirely to the decentralized decision-making processes of competitive firms. There is usually a non-market institution that has to approve, license, or provide some other imprimatur without which firms cannot change their production method. The market test by itself is not always enough. In the past, it seldom was. (Mokyr 2002, 219) 
La cita incluye la interrelación de tres elementos: los selectores (empresas y hogares), el mercado y las instituciones no mercantiles. El reconocimiento de dicha tríada se realiza junto a dos supuestos normativos y una declaración histórica. Primero, si la nueva técnica aumenta la eficiencia, entonces apoyarla debería ser una decisión un poco favorable. Segundo, las economías pueden optar por descentralizarse o no. La afirmación es que históricamente las instituciones no mercantiles han tenido el papel de aprobar cambios en el método de producción.

No obstante, me parece que una concepción de la economía política en este sentido carece de aspectos necesarios para el establecimiento de su sentido crítico con las condiciones de acumulación en las que se produce el conocimiento.

Para Marx, el método de la economía política tiene un punto de partida en el análisis de lo real y lo concreto. En los Grundrisse, Marx ( [18571858]1993) propone que la producción de conocimiento está dentro del análisis del carácter abstracto de lo que se pretende comprender: "[...] I would then, by means of further determination, move analytically towards ever more simple concepts" (Marx [1857-1858]1993, 100). En el Método de economía política (apartado incluido en Grundrisse), la abstracción es un acto analítico por el cual se producen determinaciones (abstractas) como un momento de pensamiento. En este sentido, la concepción del objeto, aunque es el punto de partida de la abstracción, constituye un momento predialéctico. Así, el pensamiento conceptual nunca debe confundirse con lo real: "The totality as it appears in the head, as a totality of thoughts, is a product of thinking head, which appropriates the world in the only way it can" (Marx [1857-1858]1993, 101). Según Ollman $(2003,13)$, "[d]ialectics restructures our thinking about reality by replacing the common-sense notion of "thing" (as something that has a history and has external connections with other things) with notions of "process" (which contains its history and possible futures) and "relation" (which contains as part of what it is its ties with other relations)".

Ir de la "cosa" al "proceso" y a la "relación" es el paso dialéctico. La idea clave es que la relación entre el trabajo y el capital, o la devaluación de la fuerza de trabajo y el imperialismo, no se da entre dos fenómenos extraños entre sí, sino que el capital funciona, de forma precisa, porque cada parte es mutuamente constitutiva de la otra en la relación misma. A partir de una 
lectura de Marx, Ollman (2003, 59-99) señala que el método dialéctico implica: 1) abstraer cosas y posiciones sociales en las relaciones que las constituyen, 2) reconocer cómo la transformación temporal de cada una implica cambios en la interconexión, 3) re-abstraer en algún nivel de generalidad, que establece patrones, tendencias o conflictos. El análisis de Marx en los Grundisse, es relevante para criticar los enfoques como el de Mokyr, que consideran el problema del conocimiento en relación con el "conocimiento útil”. De esta manera, me parece que la crítica se abre paso a la necesidad de confrontar la historia económica no-dialéctica, donde el problema de la producción de conocimiento no aparece en esta relación doble.

El problema con el argumento de Mokyr es que lo que él considera normativo es un efecto de la configuración de las relaciones de poder que no pueden ser separadas de la emergencia de las nuevas tecnologías. Además, dadas las condiciones históricas provocadas por la crisis capitalista, lo que parece ser histórico en el argumento de Mokyr es precisamente lo que debería ser problematizado. En otras palabras, si las instituciones no mercantiles tienen el papel de aprobar cambios en el método de producción, es necesario analizar el papel que dichas instituciones tienen en el proceso de acumulación.

Para Mokyr (2003, 223-224), "[t]he history of technological progress is the history of an endangered and much-resisted species... [as well as] there are instances in which the technological 'system' itself resist a novel and improved component because it does not fit the operation of the existing system”. Así como este fragmento manifiesta el espíritu de la economía política del conocimiento en la concepción de Mokyr, es posible notar sus limitaciones para el análisis de las sociedades capitalistas del siglo XXI. En este sentido, para Mokyr (2003), la "resistencia” se considera una disposición (¿natural?) que las sociedades, como resultado de intereses en conflicto, adquieren dentro de un proceso de toma de decisiones ("the existing conceptual structures in people's minds provide resistance”, 226).

El límite de esta concepción está el carácter erróneo de los sujetos del conocimiento, quienes, al mismo tiempo, son la fuerza de trabajo indispensable para su producción. Si la agencia de los sujetos se reduce a la posición de ser "selectores", la economía política del conocimiento se vería constreñida a la extensión de los cambios de juicio. Por lo tanto, su grado de éxito depende de "how strongly it is confirmed by these standards and becomes part of the 
"consensus," which is as close as any society can come to "tested" propositional knowledge” (Mokyr 2003, 227).

Hay muchos problemas relacionados con el argumento de Mokyr y la economía política del conocimiento. ¿Cómo puede estar ausente la categoría de "trabajo" en una economía política del conocimiento? Aunque el trabajo es una categoría económica, es el resultado de un proceso de construcción, tanto en el orden histórico como en el orden del pensamiento. Así, el "trabajo", como categoría abstracta, implica un ejercicio analítico que se expone en la categoría de "trabajo en general" como fruto de una "indifference towards any specific kinds of labor, presupposes a very developed totality of real kinds of labor" (Marx [1857-1858]1993, 101). En este sentido, "trabajo” es una categoría que capta una serie de determinaciones indeterminadas. Entonces, puede adquirir una verdad práctica y servir para analizar la producción histórica de conocimiento. El "trabajo" es una categoría dialéctica porque permite analizar la reproducción de la vida en diferentes tipos de sociedades (en su forma concreta), y al mismo tiempo, tiene sentido para todas las demás categorías, con las que se pueden analizar las sociedades modernas. Para Marx ([18571858]1993, 86), "production is always a particular branch of productions ... or it is a totality. But, the political economy is not technology".

Mokyr prefiere el análisis del "conocimiento útil"; sin embargo, no nos lleva a la construcción de una economía política del conocimiento pues no refleja un movimiento de lo abstracto a lo concreto. Su historia de la producción del conocimiento no es, como él pretende, una economía política (o, al menos, no retrata elementos críticos para el análisis de la producción de conocimiento en el neoliberalismo). Lo que Mokyr señala es la tensión que existe en una concepción evolucionista de la producción de conocimiento, donde la ciencia se considera una materia de consenso. Así, pasar por alto la relación dialéctica en el análisis de la economía política haría invisible que tal "consenso" se ha hecho dentro de las condiciones asimétricas de las relaciones capitalistas de producción (en esta línea, podríamos remontarnos a los períodos coloniales en el Sur Global). Precisamente, el análisis de la acumulación primitiva nos llevaría a teorizar la relación entre sujetos, mercados e instituciones no mercantiles en las diferentes crisis de la economía mundial. El hecho de que la ciencia, como forma de producción de conocimiento, haya tenido alcance en el proceso de acumulación de capital mundial, no se puede 
separar del análisis de la acumulación capitalista por desposesión de formas de conocimiento en regiones periféricas.

Lo anterior cobra relevancia ante el análisis de los cambios en la función y comercialización del conocimiento de acuerdo con las reglas de privatización del "trabajo inmaterial" (Vercellone 2007). Marx ([1867]2009, 126-194) explica el proceso de trabajo bajo el modo de producción capitalista y cómo hay aspectos de dicho proceso que han estado presentes en cada época. Este aspecto universal es la interacción entre los seres humanos y la naturaleza. En el proceso de interacción con la naturaleza, los seres humanos se preocupan por la producción de valores de uso, lo que permite que la vida laboral sea activa y creativa. Sin embargo, en el modo de producción capitalista, el proceso laboral se centra en la producción de valor (i.e., de la plusvalía). En otras palabras, el resultado de la diferencia entre el valor de la fuerza de trabajo y la duración del trabajo en un día (es decir, la jornada laboral como la duración del tiempo en que el capitalista había utilizado la fuerza de trabajo) genera el valor excedente, el resultado de un proceso de producción.

En tal proceso, los trabajadores no solo utilizan todas sus habilidades para reproducirse a sí mismos, sino también para reproducir el capital a través de un cierto período de tiempo de trabajo "no remunerado". Así, cada día de trabajo tiene una parte de "trabajo necesario" y otra parte de trabajo excedente. El proceso mediante el cual los trabajadores generan plusvalía también permite la transferencia de la depreciación de las herramientas en el producto de la mano de obra real (así, las herramientas son "capital constante"), mientras que el capital requerido para la compra de mano de obra es "capital variable".

Centrándose en las crisis de rentabilidad y en las de demanda efectiva (o crisis de subconsumo), Vakulabharanam señala que estas dos crisis están en medio del desarrollo del capitalismo. La crisis de rentabilidad fue parte de 'la larga depresión' (1873-1896), así como las crisis de demanda efectiva fue parte de ‘la gran depresión' (1929-1939). Sin embargo, la crisis que desarrolló el neoliberalismo (1973-1980) es, de nuevo, una crisis de rentabilidad, mientras que la crisis global (la gran recesión de 2008) implica una crisis subconsumista latente, en la que aún no se ha determinado el mecanismo para hacer frente a la inseguridad alimentaria y los desastres ecológicos. Este conjunto de condiciones histórico-políticas conforman lo que Vakulabharanam llama "una era de incertidumbre" que ha exigido la intensa incorporación y generación 
de subjetividades flexibles y precarias (Berardi 2012; Moulier-Boutang 2007; Federici 2013).

Así, la condición del trabajador no parece estar determinada por sus habilidades (o su condición de trabajadores "no calificados"), en parte, debido a la diversificación de las formas de producción que el capitalismo ha desarrollado. Si durante la época de Marx la fábrica era el lugar para "aprender" las habilidades necesarias para el trabajo (aunque era más bien una técnica coercitiva), hoy en día el capitalismo corporativo exige no solo el trabajo libre, sino el conocimiento social que permanece en el "potencial" del trabajador. Las "habilidades potenciales" no se aprenden necesariamente en la fábrica, sino que son un producto del origen social (cultural, intelectual o incluso étnico) del trabajador. Esta situación generaría un "capitalismo cognitivo" (Vercellone 2013; Fumagalli 2007).En él, la producción de conocimiento implica una forma de explotación más allá de la jornada laboral. Sin embargo, como señala Thiem $(2017,472)$, el tiempo de trabajo sigue siendo la sustancia del valor abstracto:

For Marx, labor time was the substance of abstract value; today originality -patentable ideas- seems more and more crucial to the sense of value. Marx's analysis also accounts for how the practices of production can at the same time heighten as well as elide our grasp of the material and immaterial inequalities that are often geographically displaced and dispersed.

A partir de Thiem (2017), es posible argumentar que la "geopolítica del conocimiento" y el "capitalismo cognitivo" proporcionan elementos teóricos que pretenden explicar cómo funciona el capitalismo del siglo XXI. Sin embargo, todavía es necesario pensar en ambas condiciones de desde una perspectiva de economía política, al menos por dos razones. Primero, porque la economía política del conocimiento establecerá elementos analíticos arraigados en la tensión entre el dominio del conocimiento y el modo de producción capitalista; $y$, segundo, para entender las formas particulares de producir conocimiento a partir de los procesos y condiciones de los actores involucrados.

La economía política del conocimiento implica no solo el análisis de los roles regionales en la geopolítica global de las economías dependientes sino de los efectos de estos roles en las posibilidades de las democracias nacionales (Coronil 1995). En otras palabras, una economía política del conocimiento 
implica abordar cómo circula el capital en el espacio y el tiempo en dos niveles relacionados dialécticamente: 1) la política entre el Estado y el Imperio, 2) los movimientos moleculares de acumulación de capital en el espacio y el tiempo.

\section{Sur Global y conocimiento en la diferencia histórica: Conexiones}

Los términos cronológicos de los períodos colonial y poscolonial en las diversas regiones del Sur Global pueden diferir, pero comparten ciertas características en la forma en que definen la relación entre la producción de conocimiento y la acumulación global. Como señala Chakravarty (2005), la formulación del capitalismo de Marx ha implicado una teleología de la historia, pues el proyecto de desarrollo de la modernidad sigue situando a las sociedades occidentales y no occidentales como dos diferentes momentos de la historia. Así, en el desarrollo del sistema capitalista, las sociedades no occidentales aparecen en un estado todavía embrionario para las sociedades occidentales: el presente de Europa es el futuro de los países del tercer mundo.

Para Chakravarty, la formulación de Marx omite que el trabajo abstracto en Europa es el resultado de la subsunción de otras formas de trabajo abstracto (que, a su vez, son el resultado de la apropiación de la fuerza laboral fuera de Europa). Al llevar este argumento al campo de la producción de conocimiento, el "conocimiento" del norte es un proceso de producción que incorpora la producción global. Solo así es posible la economía política del conocimiento: mirando al conocimiento proceso histórico global de producción.

Si bien Eric Wolf (1982) usó el marco teórico de Marx como referencia para entender las transformaciones sociales en una combinación de diferentes elementos vinculados a la reproducción de la vida (que prefieren, principalmente, la perspectiva marxista de los "modos de producción" más que la lingüística que fija significados en un juego entre símbolos), también ha criticado cómo las ciencias sociales se han propuesto explicar el mundo. De esta manera, la "historia universal" (que termina siendo el desarrollo de la razón desde Grecia hasta Alemania) ha facilitado la creación de mitos de superioridad histórica en Europa y Estados Unidos. Por ejemplo, la sociología, como disciplina separada de la economía política, constituye un objeto de conocimiento para "lo social" y, en este proceso, la posibilidad de la construcción de un "orden social” pasa a desconectarse de sus condiciones económicas, culturales e históricas. Así, la sociología evolutiva (que asume "lo urbano" como punto de llegada de las 
sociedades) encuentra en la persistencia de "la comunidad" una amenaza al "orden social".

En su análisis sobre la teoría marxista de los modos de producción, Banaji (2010) denuncia la lógica evolucionista del capitalismo desde la que se asume que la sucesión de los modos de producción (esclavitud, feudalismo, capitalismo) se extiende en el tiempo a todas las sociedades. Por lo tanto, la limitación de Marx era que no contemplaba la existencia de un tipo diferente de grupo social que funcionara como clase dominante en las sociedades coloniales, que, aunque no con las características de la burguesía europea, representaba el lugar de poder entre el campesinado y la aristocracia. No se trata de negar el esquema de funcionamiento del capitalismo (ni la teoría de los modos de producción como modelo formal para entender la dinámica histórica), sino de reconocer que determinadas configuraciones históricas son genuinas y no meramente "variantes" del él.

En este sentido, reconocer que los caminos de desarrollo del capitalismo tendrían múltiples líneas no significa una ruptura con el necesario análisis de la acumulación en cada configuración del este. Al seguir a Torres, Pannu y Bacchur (2008) se puede afirmar que:

la acumulación capitalista durante el colonialismo podría caracterizarse por la trayectoria de acumulación hacia la consolidación del Estado colonial. El orden colonial integró los sistemas precoloniales (no regulados por el Estado), con una dependencia directa de los recursos naturales, a sistemas administrativos de coerción y control que permitieron la extracción de recursos dentro de la desigualdad estructural del mercado colonial: para finalmente pretender combinar la aceleración de la educación con miras a la intensificación de extracción de recursos y la creación de mano de obra asalariada. (Vásquez, Cielo y Bravo 2016, 8-9)

Karl Marx (Capital, Vol. I, c. VIII) acuñó la idea de acumulación primitiva, que explica cómo el desarrollo del capitalismo ha implicado la transformación radical de las condiciones objetivas de la producción material a la vez que ha modifica las formas de concebir a los “trabajadores”. En cierto modo, uno de los puntos más relevantes en el análisis de Marx es cómo el sujeto de trabajo se ve afectado en este proceso de transformación. Así, el capitalismo implica una revolución en tanto alcanza todas las dimensiones de la vida 
social: la relación con la tierra que evoluciona hacia la propiedad privada, la formación del derecho como instrumento para perpetuar los crímenes contra los expropiados, la racionalización de la fuerza de trabajo para generar plusvalía, así como la regulación del salario y su establecimiento como única forma de supervivencia para los trabajadores. Entre estas variaciones, quizás la más destacada sea la forma en que la clase dominante se constituye como tal, precisamente porque domina no solo la economía sino también al Estado. Esta transformación es crítica en el análisis de la economía política del Sur Global cuando consideramos la producción de conocimiento en África y América Latina (Cielo, Vásquez y Bravo 2018).

En América Latina, el discurso neoliberal de los años ochenta y noventa consideraba al "intervencionismo estatal" y al control político de las "distorsiones del mercado" como una barrera para construir una economía de precios "reales". Esto implicó un giro hacia la autoridad del Fondo Monetario Internacional (FMI). Ambos elementos, tanto el discurso neoliberal, como el giro hacia las políticas del FMI han formado parte de las derechas políticas que alcanzaron el gobierno también en el siglo XXI. El FMI ha sido el otorgante de los préstamos destinados a asesorar a los países "para nivelar sus economías", pero al mismo tiempo exige que los Estados-nación se adapten a la competencia global en los términos diseñados en el espíritu del consenso de Washington.

Para Ferguson (2006), los ajustes estructurales que introdujo el neoliberalismo en África se obtuvieron en gran medida a través de un lenguaje que se encuentra en la legitimación de las estrategias del Banco Mundial, a través del capitalismo científico. Este se basa en la desmoralización de la economía, con la legitimidad de una "economía propia" que responde a criterios técnicos. Por lo tanto, cualquier llamado a una "economía justa” se consideraría perjudicial, para el pragmatismo necesario, encaminado al crecimiento económico.

En retrospectiva, los "ajustes estructurales" de los años ochenta funcionaron como recetas universales para países en el Sur Global. A fin de establecer la conexión entre la acumulación por desposesión y el capitalismo cognitivo en el Sur Global, es necesario investigar el sentido histórico de tres aspectos diferentes de la realidad social: el epistemológico, político y económico. Esto implica, por un lado, investigar los medios por los cuales la producción del conocimiento se legitima, así como los recursos económicos que se les asignan y, por otro lado, analizar los mecanismos de transmisión. Estos mecanismos 
responden a las condiciones o contextos sociales en los que se instala la maquinaria de la producción del conocimiento. No obstante, como señala Banaji (2010), "the capitalist system does not expand," "it does not vary from place to place”, sino que se configura de forma particular según las condiciones de cada escenario (periférico o no). A su vez, la lógica del capitalismo, como marco formal para entender la articulación de un sistema mundial, se expresa en cada una de estas particularidades, es decir, en las diversas formas de producción de conocimiento en sociedades poscoloniales bajo regímenes neoliberales.

\section{Referencias:}

Alimonda, Héctor, Catalina Toro Pérez y Facundo Martín. Ecología politica latinoamericana: Pensamiento crítico, diferencia latinoamericana y rearticulación epistémica. Buenos Aires: CLACSO, (2017).

Banaji, Jairus. Theory as History. Essays on Mode of Production and Exploitation. Londres: Brill, (2010).

Chakravarty, Dipesh. Provincializing Europe: Postcolonial Thought and Historical Difference. Princeton: Princeton University Press, (2005).

Chatterjee, Partha. "Land and the Political Management of Primitive Accumulation". En The Land Question in India: State, Dispossession and Capitalist Transition, editado por Anthony P. D’Costa y Archin Chakraborty, 1-15. Oxford: Oxford University Press, (2016).

Cielo, Cristina, Jorge Daniel Vásquez y Pedro Bravo. "A situated political economy of knowledge: Critical Social Sciences in Tanzania and Ecuador", The International Education Journal. Comparative Perspectives. 17 (4), 49-67, (2018).

Coronil, Fernando. "Transculturation and the Politics of Theory: Countering the Center, Cuban Counterpoint”. En Cuban Counterpoint: Tobacco and Sugar, de Fernando Ortiz, ix-lvi. Durham: Duke University Press, (1995).

Ferguson, James. "Transnational topographies of power: Beyond 'the State' and 'Civil Society' in the Study of African Politics”. En Global Shadows: Africa in the Neoliberal World Order. Durham: Duke University Press, (2006).

Fumagalli, Andrea. Bioeconomía y capitalismo cognitivo. Hacia un nuevo paradigma de la acumulación. Madrid: Traficantes de Sueños, (2007).

Harvey, David. The Limits to Capital. Chicago: University of Chicago Press, (1982).

Harvey, David. The New Imperialism. Oxford: Oxford University Press, (2003).

Luxemburgo, Rosa. The Accumulation of Capital. Londres: Routledge and Kegan Paul Ltd, ([1913]1951.

Marx, Karl. Grundrisse. Foundations of the Critique of Political Economy (Rough Draft). Traducido por M. Nicolaus. Londres: Penguin Books, ([1857-1858]1993).

Marx, Karl. Capital. A Critique of Political Economy. Vol. 1. Nueva York: International Publishers, ([1857]2009).

Meyer, John W., y Francisco O. Ramírez. La educación en la sociedad mundial: Teoría institucional y agenda de investigación de los sistemas educativos contemporáneos. Barcelona: Octaedro. 
Mokyr, Joel. (2002). The Gifts of Athena. Historical Origins of the Knowledge Economy. Princeton: Princeton University Press, (2010).

Ollman, Bertell. Dance of the Dialectic: Steps in Marx's Method. Chicago: University of Illinois Press, (2003).

Panizza, Francisco. Contemporary Latin America. Development and Democracy beyond the Washington Consensus. Londres: Zed Books, (2009).

Thiem, Yannik. "Critical Theory in the Age of Knowledge Capitalism: Elusive Exploitation, Affects, and New Political Economies". The Journal of Speculative Philosophy 31 (3): 468-480, (2017).

Tijoux, María Emilia. Naturaleza americana: Extractivismo y geopolítica del Capital. Santiago de Chile: LOM, (2016).

Torres, Carlos Alberto, Rajinder Pannu y Kazim Bacchur. "Acumulación de capital, legitimación política y expansión educativa”. En Sociología política de la educación en perspectiva internacional y comparada, compilado por Carlos Mora-Nici y Guillermo Ruiz. Buenos Aires: Miño y Dávila Editores, (2008).

Vakulabharanam, Vamsi. "Economic Turbulence in Capitalism: Twentieth Century and Beyond". En Marxism with and beyond Marx, editado por Amiya Kumar Bagchi y Amita Chatterjee, 115-135. Delhi: Routledge, (2014).

Vakulabharanam, Vamsi. Rereading Das Kapital in the 21st Century. Economic EO Political Weekly 52 (37), 42-50, (2017).

Vásquez, Jorge Daniel, Cristina Cielo y Pedro Bravo. Economía política del conocimiento en el sur global: el caso de la educación superior en Ecuador y Tanzania. Buenos Aires: CLACSO [Informe de Investigación], (2016).

Vercellone, Carlo. "From Formal Subsumption to General Intellect: Elements for a Marxist Reading of the Thesis of Cognitive Capitalism, in Historial Materialism”. Historial Materialism 15 (1): 13-36, (2007).

Vercellone, Carlo. "Capitalismo Cognitivo. Releer la economía del conocimiento desde el antagonismo capital-trabajo”. Traducido por Ignacio Paz. Ponencia de la conferencia pronunciada en la Universidad de San Martin, Buenos Aires, (2013).

Wolf, Eric. Europe and the People Without History. Berkeley: University of California Press, (1982). 